\title{
Complex middle cerebral artery aneurysms: a new classification based on the angioarchitecture and surgical strategies
}

\author{
Wei Zhu • Peixi Liu • Yanlong Tian • Yuxiang Gu • \\ Bin Xu • Liang Chen $\cdot$ Liangfu Zhou $\cdot$ Ying Mao
}

Received: 21 March 2013 / Accepted: 29 April 2013 /Published online: 30 May 2013

(C) The Author(s) 2013. This article is published with open access at Springerlink.com

\begin{abstract}
Background Because of the diversity of aneurysm morphology, complicated arterial anatomy and hemodynamic characteristics, tailored surgical treatments are required for cases of individual complex middle cerebral artery (MCA) aneurysms.

Methods During an 8-year period, 59 complex MCA aneurysms in 58 patients were treated microsurgically in our department. Complex aneurysms were defined as having large (10-24 $\mathrm{mm}$ in diameter) or giant (diameter $\geq 25 \mathrm{~mm}$ ) size or non-saccular morphology (fusiform, dissecting or serpentine). Results Direct clipping of the aneurysmal necks was achieved in eight patients, while reconstructive clipping was performed in 25 patients. Indirect aneurysm occlusion was performed in 25 cases, including trapping or resecting the aneurysm in four cases, trapping or resecting the aneurysm with extraintracranial (EC) or intra-intracranial (IC) bypass in 21 cases and internal carotid artery (ICA) sacrifice with EC-IC bypass in one case. Forty-eight aneurysms $(81.4 \%)$ were completely obliterated. Graft patency was confirmed in 20 of 21 cases $(95.2 \%)$ with bypass. A recurrent aneurysm was detected in one case and a re-operation was performed. Two patients with Hunt-Hess grade IV aneurysms died during the perioperative period. Overall, 52 cases $(88.1 \%)$ had good outcomes (Glasgow Outcome Scale $\geq 4$ ) during the late follow-up period.
\end{abstract}

Wei Zhu and Peixi Liu contributed equally to this article.

W. Zhu $\cdot$ P. Liu $\cdot$ Y. Tian $\cdot$ Y. Gu $\cdot$ B. Xu $\cdot$ L. Chen $\cdot$ L. Zhou $(\bowtie) \cdot$

Y. Mao $(\bowtie)$

Department of Neurosurgery, Huashan Hospital of Fudan

University, 12 Wulumuqi zhong Road,

Shanghai 200040, China

e-mail: 1fzhouc@online.sh.cn

Y. Mao

e-mail: maoying@fudan.edu.cn
Conclusion The surgical modality and strategy for treating complex MCA aneurysm are decided according to the morphology of the aneurysm, vascular anatomy and the hemodynamic characteristics of each case. Thus, we developed a new classification based on the angioarchitecture. Favorable outcomes can be achieved by treating complex MCA aneurysms with appropriate surgical modalities, strategies and techniques.

Keywords Middle cerebral artery · Complex aneurysm . Bypass surgery $\cdot$ Clipping

\section{Introduction}

Patients with complex middle cerebral artery aneurysms may present with intracranial hemorrhage, mass effects, epilepsy or cerebral ischemia; in addition, the aneurysm may be incidentally discovered. A high mortality rate of 65-85\% within 2 years (because of rupture or re-rupture) has been reported [7-10], and aneurysm rupture survivors are often left with severe neurological deficits.

Complex middle cerebral artery aneurysms include aneurysms that are sizeable (large or giant), broad-necked, fusiform, dissecting or serpentine; many of these types of aneurysms cannot be treated with direct aneurysm neck clipping or dome coiling. Although wrapping the aneurysm may decrease the bleeding risk, it is not really effective for complex aneurysms.

Because of the diversity of aneurysm morphology and the location and hemodynamic differences among patients, tailored surgical treatment is required for each individual case. Techniques such as aneurysm trapping or resection and arterial reconstruction play a pivotal role in treating these formidable lesions. This article reports on our surgical strategies for treating these difficult lesions. 


\section{Materials and methods}

This study was approved by the Ethics Committee of Huashan Hospital, Fudan University. Complex aneurysms were defined as having a large (10-24 $\mathrm{mm}$ in diameter) or giant (diameter $\geq 25 \mathrm{~mm}$ ) size or non-saccular morphology (fusiform, dissecting or serpentine). During an 8-year period from March 2004 to July 2012, 59 complex middle cerebral artery (MCA) aneurysms in 58 patients were treated microsurgically and followed up in our department. The patients included 30 men and 28 women with a mean age of 47 years (range 19-66 years). Twentyseven patients presented with subarachnoid hemorrhages or intracerebral hematomas. The Hunt-Hess grades were as follows: nine patients had grade I, eight patients grade II, four patients grade III and six patients grade IV. Eight patients harbored multiple aneurysms at different sites. Two patients presented with recurrent aneurysms after previous endovascular coiling, and one patient presented with a recurrent aneurysm after previous microsurgical clipping. Aneurysm locations and morphologies are listed in Tables 1 and 2.

The treatment strategies for each case were decided by a combined cerebrovascular team that comprised both neurovascular surgeons and endovascular specialists. The exclusion criteria included a Hunt-Hess grade $\mathrm{V}$ presentation, aneurysm calcification, advanced age and significant anesthetic or surgical risks. Surgical modalities included direct clipping or reconstructive clipping of the aneurysmal neck, aneurysm resection and parent artery occlusion, with or without bypass. The adequacy of the treatment and patency of the parent vessels were analyzed intraoperatively using indocyanine green fluorescence angiography and/or Doppler flow measurements. Intraoperative sensory-evoked potential (SEP) monitoring and motor-evoked potential (MEP) monitoring were routinely performed to detect potential injury to neurological functions.
All preoperative and postoperative clinical information, neurologic examinations and imaging data, including computed tomography, magnetic resonance imaging and cerebral angiography, were reviewed retrospectively. Follow-up information from all patients was obtained by telephone or clinical interview, and the most current Glasgow Outcome Scale (GOS) score was reported for each patient. The patency of the graft was followed by 3D CT angiography, magnetic resonance angiography (MRA) or digital subtraction angiography for 6 months postoperatively. Subsequently, 3D CT angiography was performed every 1 or 2 years thereafter. The average clinical follow-up time was 38 (range 3-97) months; recent follow-ups were performed by clinic interview in 42 cases and by phone in 16 of the total 58 cases. The average radiological follow-up period was 32 (range 3-70) months.

\section{Results}

\section{Surgical treatment}

Direct clipping of the aneurysmal necks was achieved in eight patients, while reconstructive clipping after temporary trapping or a thrombectomy was performed in 25 patients. Indirect aneurysm occlusion was performed in 25 cases, including trapping or resection of the aneurysm in four cases, trapping or resection of the aneurysm with extra-intracranial or intraintracranial bypass in 21 cases and ICA sacrifice with EC-IC bypass in one case (Tables 1 and 3).

During the operation, brain relaxation was achieved by administration of mannitol $(1 \mathrm{~g} / \mathrm{kg})$ and dexamethasone $(5 \mathrm{mg})$ or cerebrospinal fluid drainage through dissection into a subarachnoid cistern. During the anastomosis when the parent artery was temporary occluded, mild hypothermia, a barbiturate or propofol was used to increase tolerance to ischemia. Twelve cases experienced an intraoperative MEP signal decrease during temporary

Table 1 Aneurysms locations, surgical modalities and the classification

\begin{tabular}{|c|c|c|c|c|c|c|c|}
\hline \multirow[t]{2}{*}{ Classification } & \multirow{2}{*}{$\begin{array}{l}\text { Aneurysm } \\
\text { location }\end{array}$} & \multirow[t]{2}{*}{$\mathrm{n}$} & \multirow[t]{2}{*}{$\%$} & \multicolumn{4}{|l|}{ Surgical modality } \\
\hline & & & & $\begin{array}{l}\text { Direct or } \\
\text { constructive clip }\end{array}$ & $\begin{array}{l}\text { Aneurysm } \\
\text { trapping/ } \\
\text { resection }\end{array}$ & $\begin{array}{l}\text { Aneurysm } \\
\text { trapping/ } \\
\text { resection+bypass }\end{array}$ & $\begin{array}{l}\text { ICA } \\
\text { sacrifice }+ \\
\text { bypass }\end{array}$ \\
\hline Type A & M1 & 7 & 12 & 4 & & 2 & 1 \\
\hline Type B & $\begin{array}{l}\text { M1-M2 bifurcation } \\
\text { M2 }\end{array}$ & $\begin{array}{l}28 \\
8\end{array}$ & $\begin{array}{l}47 \\
14\end{array}$ & 19 & & 17 & \\
\hline Type C & $\begin{array}{l}\text { M2-M3 bifurcation } \\
\text { M3 and distal }\end{array}$ & $\begin{array}{l}12 \\
4\end{array}$ & $\begin{array}{l}20 \\
7\end{array}$ & 10 & 4 & 2 & \\
\hline Total (n) & & 59 & 100 & 33 & 4 & 21 & 1 \\
\hline
\end{tabular}


Table 2 Morphology

of Aneurysms

\begin{tabular}{ll}
\hline Morphology & $\mathrm{n}$ \\
\hline Giant or large & 57 \\
Fusiform & 25 \\
Dissecting or serpentine & 23 \\
\hline
\end{tabular}

parent artery occlusion. Changes in SEP or MEP were managed by increasing the blood pressure with pressor agents. The waves returned to normal in all except four cases after revascularization or relief of temporary clipping. These four patients presented with temporary or permanent neurological deficits due to perforator injury or MCA territory ischemia. Systemic Dextran 40 and heparin irrigation in the surgical field were used during the anastomosis.

\section{Aneurysm outcomes}

Forty-eight aneurysms $(81 \%)$ were completely occluded with no residual aneurysm or neck remnant. Eleven aneurysms (19\%) had a minimal residual aneurysm after clipping, and further treatment was deemed unnecessary. Graft patency was confirmed in 20 of 21 cases with bypass. No rebleeding was found in any cases during the follow-up period. A recurrent aneurysm was detected in one case during the 2-year follow-up period, and a re-operation was performed.

\section{Patient outcomes}

Two patients with Hunt-Hess grade IV died during the perioperative period; both patients presented severe aneurysmal intracerebral hemorrhage (ICH) and required emergency surgery. Two patients died from nonsurgical-related diseases during the late follow-up period. Five patients presented with cerebral ischemia, including one MCA territory infarction caused by a bypass occlusion and four focal ischemic deficits caused by injury to the perforators. Two patients developed ICH after surgery, including one who required a hematoma evacuation. Meningitis occurred in

Table 3 Surgical management of complex MCA Aneurysms

\begin{tabular}{lll}
\hline Surgical treatment & $\mathrm{n}$ & $\%$ \\
\hline Direct clipping & 8 & 13.6 \\
Clip reconstruction of the aneurysmal neck & 25 & 42.4 \\
Aneurysm trapping/resection & 4 & 6.8 \\
Aneurysm trapping/resection+EC-IC bypass & 16 & 27.1 \\
Aneurysm trapping/resection+IC-IC bypass & 5 & 8.5 \\
ICA sacrifice+EC-IC bypass & 1 & 1.7 \\
Total & 59 & 100 \\
\hline
\end{tabular}

three patients, and all of them were cured with antibiotics. Two patients experienced seizures that were controlled with medication.

The preoperative and postoperative GOS scores of all the patients are listed in Table 4. Fifty-two cases had good outcomes (GOS 5 or 4) during the late follow-up period, and a total of four patients died. In general, the patients with unruptured aneurysms were more likely to have favorable outcomes than the subarachnoid hemorrhage (SAH) patients.

Illustrative cases

Case 1: (Fig. 1)

A 19-year-old man suffered from a sudden severe headache and became unconsciousness $2 \mathrm{~h}$ before admission. A physical examination showed a Glasgow Coma Scale (GCS) score of 6, and the right pupil was dilated. A computed tomography (CT) scan revealed SAH and a large hematoma in the right temporal lobe. The emergent $\mathrm{CT}$ angiogram demonstrated a giant ruptured aneurysm originating from the M1 segment. Open surgery was conducted emergently. After evacuating the majority of the hematoma, the aneurysm was exposed. The lenticulostriate perforators from M1 were meticulously identified. After we confirmed that these perforators were not involved in the neck of the aneurysm, the aneurysm was clipped using a neck reconstruction technique. The patient recovered quickly after surgery, and the GOS score reached 5 at the 3-month follow-up appointment. A postoperative CT angiography (CTA) demonstrated complete obliteration of the aneurysm.

The recurrence of the aneurysm was detected by CTA at the 1-year appointment and confirmed at the 2-year followup appointment; it was determined that reoperation was required. Intraoperative findings showed a dissecting aneurysm with severe calcification of the aneurysmal wall, which prevented direct aneurysm clipping. Fortunately, the lenticulostriate perforators did not originate from the site

Table 4 Patient outcomes after surgical intervention

\begin{tabular}{|c|c|c|c|c|c|c|}
\hline \multirow[t]{2}{*}{ GOS } & \multicolumn{2}{|c|}{ Preoperation } & \multicolumn{2}{|c|}{ Early postoperation } & \multicolumn{2}{|c|}{ Late follow-up } \\
\hline & $\mathrm{n}$ & $\%$ & $\mathrm{n}$ & $\%$ & $\mathrm{n}$ & $\%$ \\
\hline 5 & 30 & 50.8 & 38 & 64.4 & 46 & 78.0 \\
\hline 4 & 16 & 27.1 & 9 & 15.3 & 6 & 10.2 \\
\hline 3 & 7 & 11.9 & 8 & 13.6 & 3 & 5.1 \\
\hline 2 & 6 & 10.2 & 2 & 3.4 & 0 & 0 \\
\hline 1 & 0 & 0 & 2 & 3.4 & 4 & 6.8 \\
\hline Total & 59 & 100 & 59 & 100 & 59 & 100 \\
\hline
\end{tabular}


Fig. 1 Case 1. a The CTA revealed a giant aneurysm originating from the right M1 segment in this 19-year-old man. b The CTA showed complete obliteration of the aneurysm after the first surgery. c Regrowth of the aneurysm was detected on the CTA at the 1-year follow-up appointment. d The recurrent aneurysm was enlarged at the 2-year follow-up appointment. e The DSA confirmed the aneurysm recurrence before the second surgery. f Intraoperative findings revealed a dissecting aneurysm (*) with severe calcification of the aneurysmal wall, which prevented direct aneurysm clipping. g A diagram of the intraoperative findings shows the recurrent aneurysm originating from the proximal M1 segment. $\mathbf{h}$ The diagram shows that the recurrent aneurysm was trapped without injury to the lenticulostriate perforators. i An intraoperative photo showed the trapping of the recurrent aneurysm (*). $\mathbf{j}$ STA (arrowhead)-M3 (arrow) bypass. $\mathbf{k}$ The postoperative CTA showed that the aneurysm was obliterated, and the bypass was patent
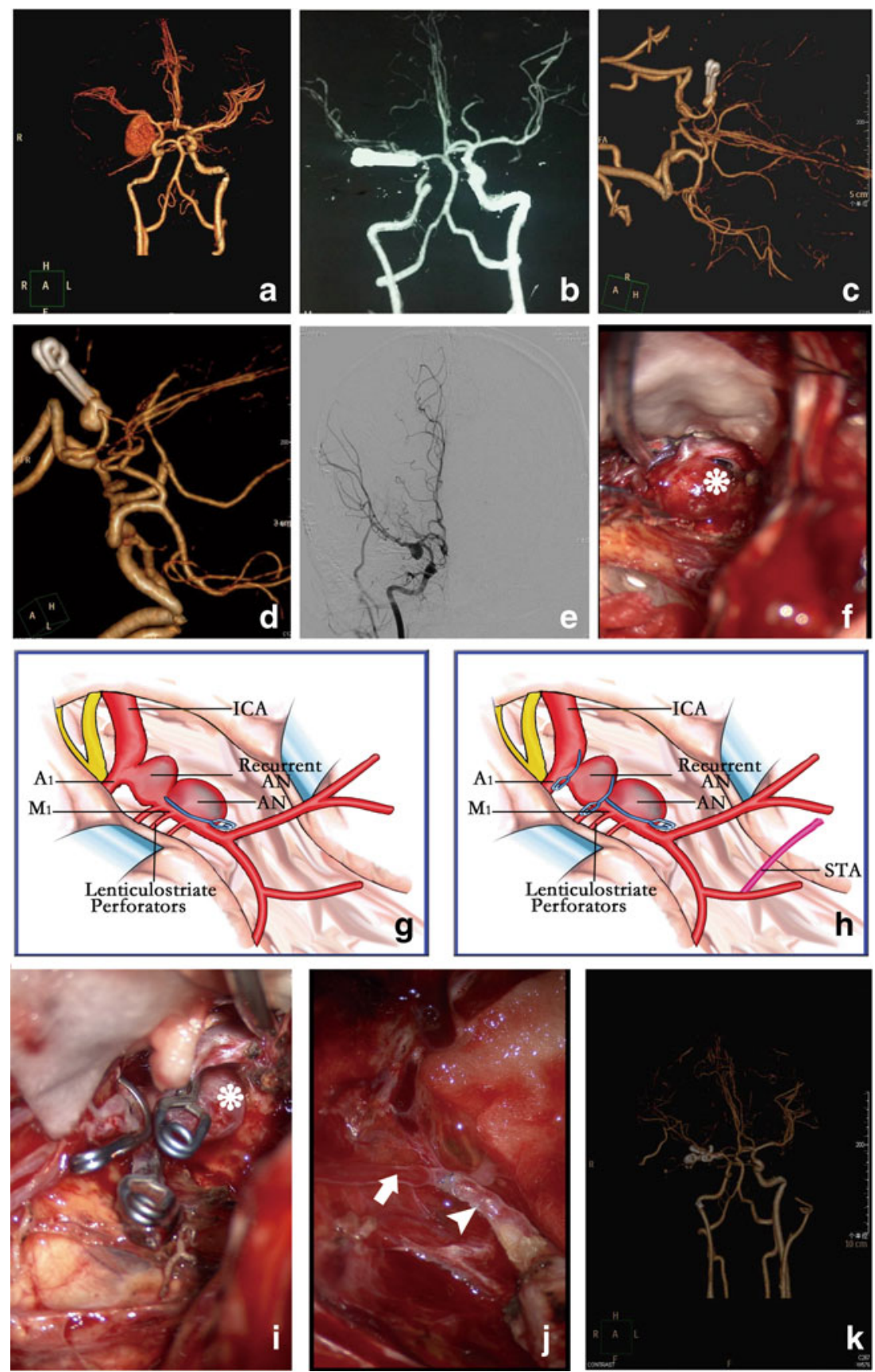

of the aneurysm location. Therefore, the aneurysm was trapped after a superficial temporal artery (STA)-M3 bypass had been performed. The patient recovered without any neurological deficits. The postoperative CTA demonstrated complete obliteration of the aneurysm and patency of the bypass graft.

\section{Case 2: (Fig. 2)}

A 56-year-old man presented with a headache and aphasia for $3 \mathrm{~h}$. A CT scan in a local hospital showed a giant lesion in the left frontal lobe. After the lesion had been diagnosed as a giant unruptured and thrombotic MCA aneurysm, it was partially coiled. The patient was transferred to our department. The Digital Subtraction Angiography (DSA) revealed a giant serpentine aneurysm originating from the M1 segment, and direct clipping or further endovascular treatment was impossible. Furthermore, it would have been unavoidable to sacrifice the lenticulostriate perforators if we had trapped the aneurysm. Therefore, a left STA-RA-M3 bypass followed by a staged left cervical ICA occlusion was performed; the ICA was finally occluded after 7 days. The postoperative DSA demonstrated that the aneurysm was completely obliterated; the ICA was sacrificed, and the MCA territory was supplied by a bypass 
Fig. 2 Case 2. a The CT

showed a partially coiled giant aneurysm in a 56-year-old man. b The MRI revealed a giant serpentine aneurysm. c-e The DSA confirmed that the aneurysm originated from the left M1 segment. f-g The diagram shows the surgical procedure. Intraoperative findings showed that the lenticulostriate perforators were involved. The cervical ICA was sacrificed via staged occlusion after a left STA-RA-M3 bypass. The aneurysm was obliterated after complete intraluminal thrombosis, and the blood flow in the MCA territory was supplied by an STA-RA-M3 bypass. The lenticulostriate perforators were occluded in a prolonged fashion. h-k A postoperative angiography demonstrated obliteration of the aneurysm. h Right ICA angiogram. i A left CCA angiogram showed occlusion of the left IC. j A left CCA angiogram showed the left MCA territory was supplied by the STA-RA-M3 bypass.

$\mathbf{k}$ Left VA angiogram
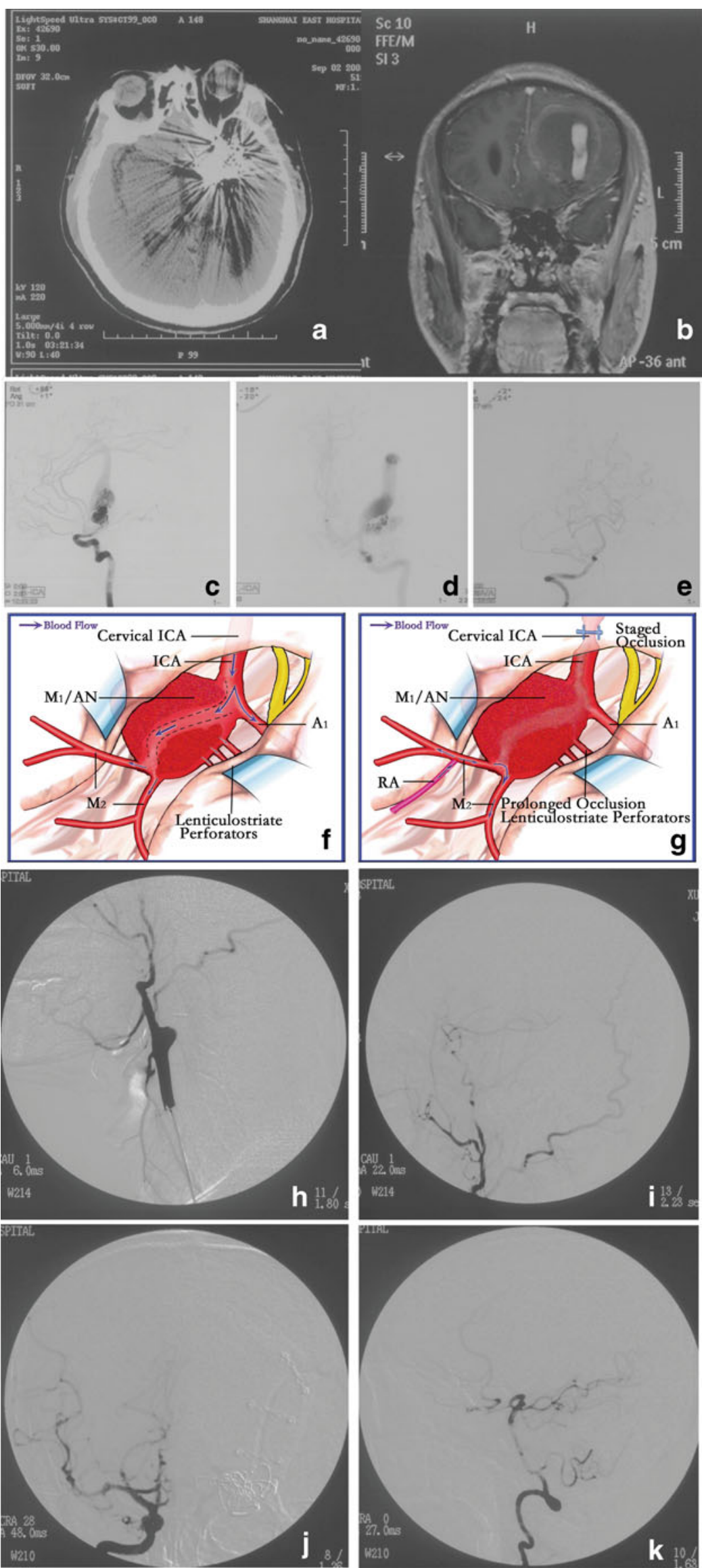
graft from STA. The patient did not present with any neurological deficits at the 3-month and 2-year follow-up appointments.

\section{Case 3: (Fig. 3)}

A 28-year-old woman presented with a headache. A CT scan revealed a giant lesion in the left front-temporal lobe. A DSA confirmed it was a giant serpentine aneurysm originating from the M2 segment. Open surgery was required. The aneurysm was exposed using the left pterional approach, and a STA-RAM3 bypass was performed. After confirming the graft patency, the proximal and distal M2 segments were temporary occluded. Intraoperative MEP and SEP showed no change after $30 \mathrm{~min}$ of occlusion. Therefore, the aneurysm was trapped and eventually resected. The patient recovered well without any neurological deficits. The postoperative CTA showed that the aneurysm had been completely obliterated, and the graft was patent.

\section{Case 4: (Fig. 4)}

A 45-year-old man presented with a headache. The CT and MRI scans showed a large lesion in the right sylvian fissure, and a partially thrombosed giant aneurysm was suspected. The DSA confirmed the diagnosis as a giant right MCA aneurysm originating from the M3 segment. During the surgery, it was found to be an unclippable aneurysm because of its dissecting morphology. Therefore, the proximal parent artery was temporary clipped under MEP monitoring. No MEP change was detected during the 30-min monitoring period. Therefore, the aneurysm was treated with a simple clipping on the proximal segment of M3. The patient recovered from the surgery without any neurological deficits. The postoperative CTA and DSA showed complete obliteration of the aneurysm.

\section{Discussion}

Surgical strategies and modalities for treating complex MCA aneurysms (Fig. 5)

Because of the diversity of aneurysm morphology, locations and hemodynamic features, complex MCA aneurysms need

Fig. 3 Case 3. a-b The DSA revealed a giant dissecting aneurysm originating from the left M2 segment in this 28 -year-old woman. c-d A diagram shows the intraoperative procedure. The aneurysm was trapped after a left STA-RA-M3 bypass had been performed. e-f Intraoperative photos showed the aneurysm (*) was trapped and resected after the bypass (arrow head) had been performed. g Intraoperative MEP monitoring showed that no change occurred during the procedure. h-i A postoperative CTA showed that the graft was patent, and the aneurysm was completely obliterated tailored treatment. The irregular morphology, giant size, intraluminal thrombus, calcified aneurysm wall, locations with
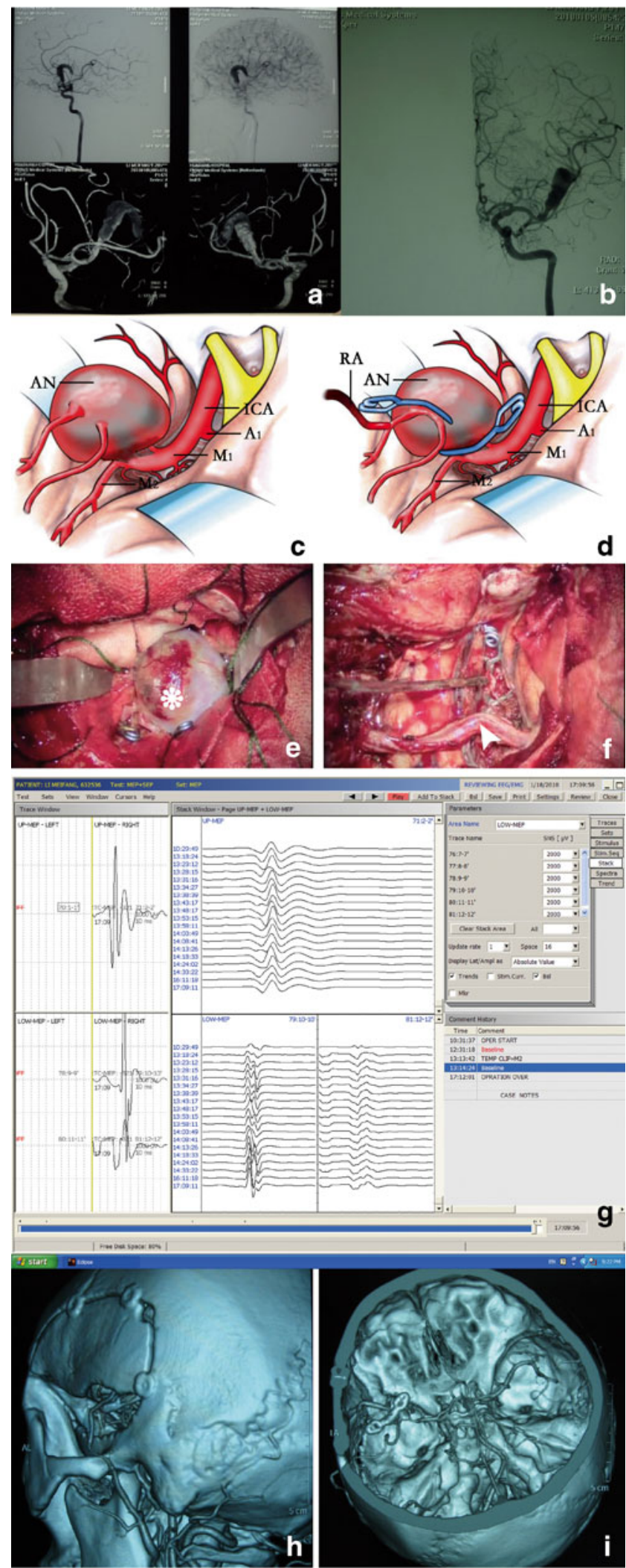
Fig. 4 Case 4. a-d CT, MRI and DSA showed a giant dissecting aneurysm originating from the right M3 segment in this 45-yearold man. e-f A diagram shows the intraoperative procedure. The aneurysm was occluded via clipping on the proximal M3 segment. g An intraoperative photo showed that the aneurysm (*) originated from the M3 segment (arrow). h An intraoperative photo showed the aneurysm $(*)$ was treated by clipping the proximal M3 segment. $\mathbf{i}-\mathbf{j}$ Intraoperative SEP (i) and MEP (j) monitoring showed no change during the surgical procedure. $\mathbf{K}-\mathbf{N}$

Postoperative images showed the obliteration of the aneurysm. CT (k), CT angiogram (l), DSA (m-n)
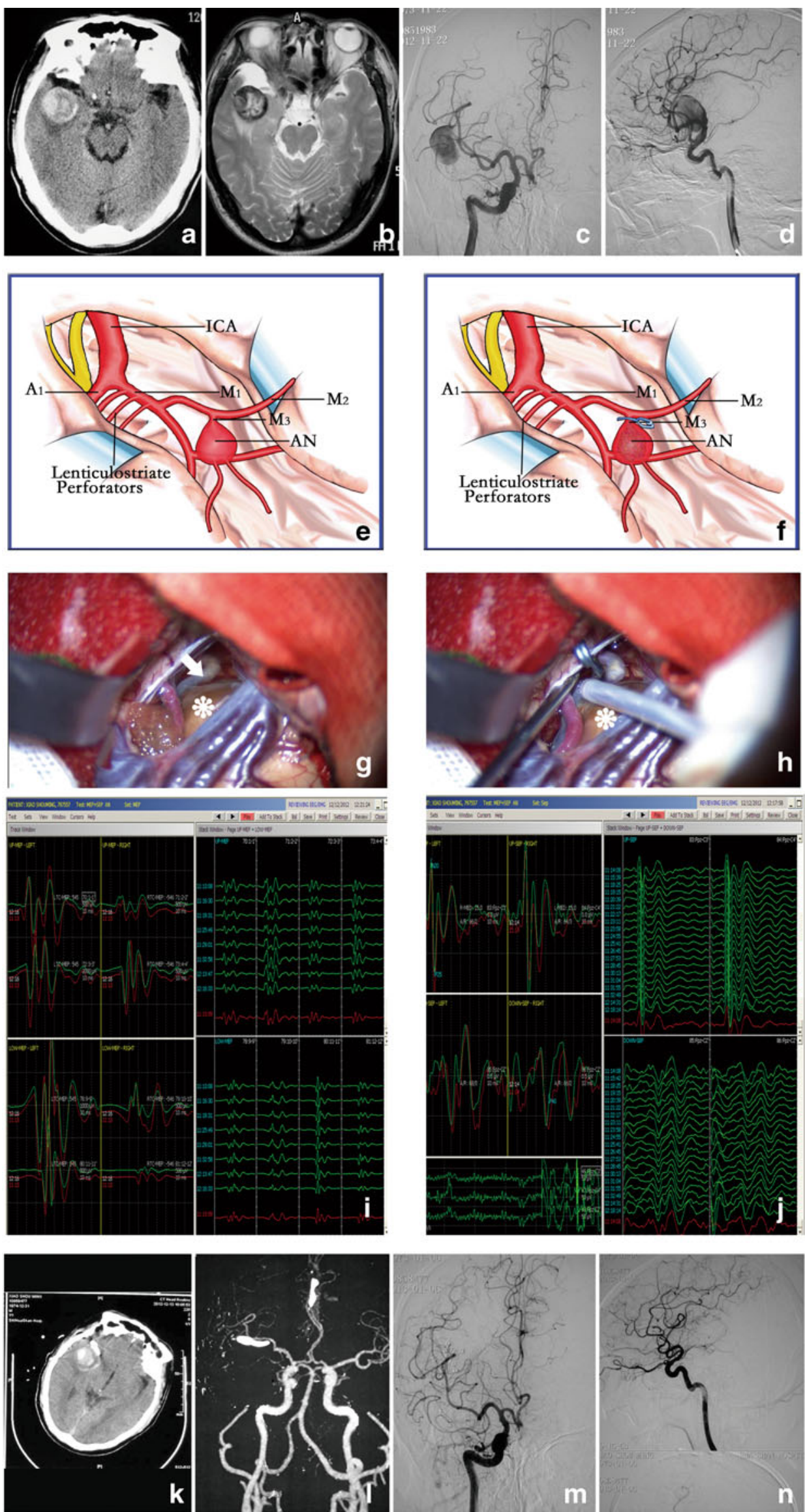


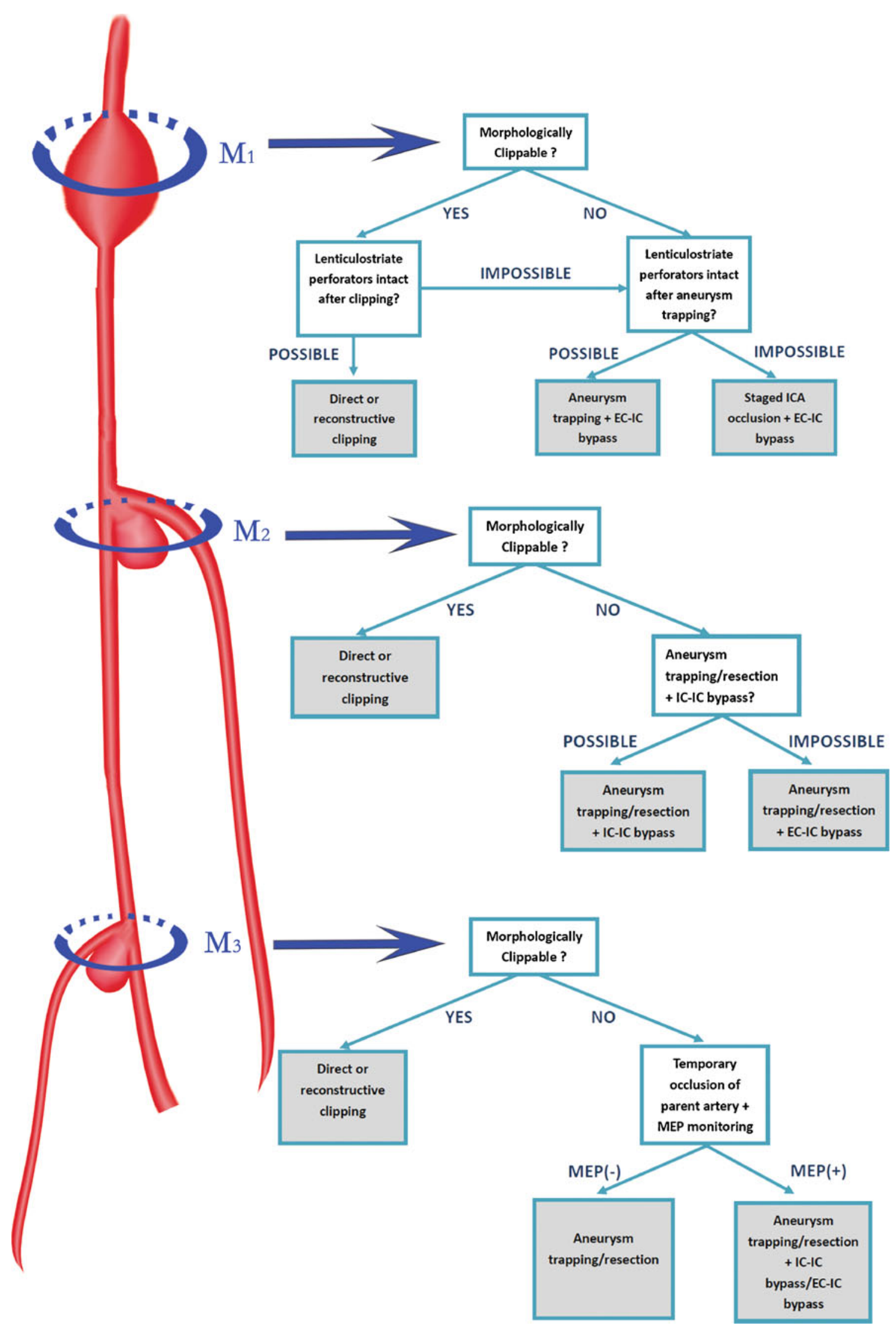

Fig. 5 The algorithm of our surgical strategies for treating complex middle cerebral artery aneurysms 
complicated vascular anatomy and hemodynamic characteristics often make the treatment extremely difficult.

Among the surgical techniques, direct neck clipping is always the preferred option when it is technically achievable $[13,19]$. For saccular aneurysms with a broad neck or giant size, we often use low-flow electrocoagulation to shrink the aneurysm wall or to mold the neck after temporary parent artery clipping. This procedure is minor, but it may transform an unclippable aneurysm into a clippable one. For giant aneurysms with severe intraluminal thrombus, a thrombectomy is usually required to facilitate direct clipping [20]. For previously coiled aneurysms, an evacuation of the coils under temporary parent artery occlusion is usually performed before we can apply a clip on the neck of the aneurysm [15, 22]. In this series of cases with complex MCA aneurysms, only eight (14\%) patients were treated using direct clipping. Twenty-five cases were managed with reconstructive neck clipping, which is a commonly used surgical technique for complex aneurysms [4, 11, 14, 17, 23]. However, this technique is only suitable for broad-necked aneurysms without atherosclerotic necks or for some fusiform aneurysms in which the parent artery can be reconstructed with clips. Aneurysms treated with reconstructive neck clipping may achieve immediate angiographic obliteration, but this procedure could lead to a high recurrence rate. Therefore, this technique is often combined with aneurysm wrapping to reinforce the reconstructed wall of the parent artery $[5,12]$.

Direct or reconstructive neck clipping is often prevented by dolichoectatic morphology, aberrant branch anatomy and atherosclerotic aneurysm necks in the majority of the complex ACA aneurysm cases. In this situation, indirect aneurysm occlusion with or without a bypass may become a unique method in many cases, with indirect occlusion consisting of proximal occlusion, distal occlusion or trapping. We had 25 patients who were treated with indirect clipping, 20 of whom required bypasses. The modality of bypass was chosen based on the vascular anatomy and hemodynamic changes after the aneurysm had been occluded. We preferred in-situ anastomosis, which could be simply and quickly performed without destroying other vascular structures. When this procedure was unachievable, STA-MCA or STA-RA-MCA would be conducted. These modalities of bypass could be performed with a predictable time of ischemia, and these procedures were not technically challenging for experienced surgeons. In our series of cases, the patency of bypass grafts was confirmed in 20 of 21 cases.

Surgical strategies for treating unclippable MCA aneurysms and the new classification

The main concerns in surgical planning include the location and morphology of the aneurysm, involvement of branch arteries, especially the lenticulostriate perforators, the potential hemodynamic changes after aneurysm obliteration and the possibility and type of arterial reconstruction. The intraoperative hemodynamic and electrophysiological assessment plays an important role in predicting the possibility of surgery-related cerebral ischemia.

Surgical planning is mainly based on the origin and morphology of the aneurysm. In this study, we classified the complex MCA aneurysms into three types. In type $\mathrm{A}$, the aneurysm originated from the M1 segment. In type B, the aneurysm originated from the M2 segment or MCA bifurcation, and in type $\mathrm{C}$, the aneurysm originated from the $\mathrm{M} 3$ or distal segments.

The main difficulty in treating type A aneurysms is preserving the lenticulostriate perforators, which are often incorporated into the aneurysm base or dome. Direct clipping of the aneurysm neck is the superior surgical modality when the perforators are not involved in the aneurysm neck and can be preserved after applying the clips. In other situations, direct clipping or trapping may lead to unavoidable damage to these perforators even if distal blood flow of the aneurysm is reconstructed. Seo and colleagues [18] reported on a patient with an M1 complex aneurysm who underwent aneurysm trapping and an STA-SV-M2 bypass. A postoperative cerebral angiogram showed ischemic infarction in the internal capsule that was consistent with lenticulostriate artery occlusion. The patient suffered from hemiparesis. Doormad et al. [21] reported on 22 patients with complex MCA aneurysms who were treated with the Excimer Laser-assisted Nonocclusive Anastomosis technique. Twenty of the aneurysms originated from the M1 segment and were treated with aneurysm trapping or resection combined with an M1-M3 ELANA flow replacement bypass. The surgical-related ischemia rate was $27 \%$. In this situation, injury to the lenticulostriate perforators was unavoidable with aneurysm neck clipping or trapping; therefore, we advocated for using STA-RA-MCA bypass followed by ICA sacrifice. This surgical modality may not achieve acute aneurysm obliteration, but it could significantly decrease the amount of blood filling the aneurysm. These postsurgical hemodynamics reduce the risk of aneurysm rupture and initiate aneurysm thrombosis, which is considered protective. Although thrombosis initiated by bypass and staged ICA occlusion can also potentially occlude the lenticulostriate perforators originating from the aneurysm, this delayed occlusion of perforators allows the involved neurons to tolerate the ischemia or promotes the construction of potential lateral circulation. In illustrative case 2 , the patient did not present with any surgical-related ischemia, and the cerebral angiogram taken on the 14th day after the operation demonstrated complete aneurysm obliteration and graft patency.

We used an STA-RA-MCA bypass (a type of vessel reconstruction) to treat the patients with type A aneurysms. Instead of a conventional high-flow EC-IC bypass (ECARA-MCA or ECA-SV-MCA), an STA-RA-MCA bypass 
has some obvious advantages [1], such as needing a shorter interpositional graft and avoiding an additional cervical skin incision. Although we did not quantitate the blood flow through the STA-RA-M2 bypass graft, Alaraj et al. [2] reported a high flow-carrying capacity of the STA trunk $(54-100 \mathrm{ml} / \mathrm{min}$ ), and the flow through the radial artery as the graft has been shown to range from $50-70 \mathrm{ml} / \mathrm{min}$. This blood flow is sufficient to support the circulation in the area of the MCA in which normal blood flow is approximately $250 \mathrm{ml} / \mathrm{min}$ above the ischemic threshold. ICA occlusion can be achieved by endovascularly placing a balloon or by staged clipping of the cervical ICA with a Silverstone clamp.

In treating type B aneurysm cases, if sacrifice of the parent artery was necessary to obliterate the aneurysm, an arterial reconstruction, such as an STA-RA-M3 or intracranial M3M3 bypass, should be conducted before the aneurysm is trapped. The aneurysm was exposed via a trans-pterional trans-sylvian approach. The region of the aneurysm was carefully inspected to identify any branches that might have been dropped over the aneurysm and also any branches or perforators that might have been compromised by a clip application or aneurysm resection. Dissection of the surrounding brain tissue is usually required to expose the branches below the aneurysm dome. After the M1, two of the M2 branches and the aneurysm were visualized, and the clip and bypass planning was determined. One of the M3 branches that was distal to the parent artery was chosen as the recipient artery for the bypass.

In some reports, patients who underwent one branch of M2 occlusion without a bypass occasionally did not present with postoperative cerebral ischemia. However, there is no accurate predictor for cerebral ischemia after an M2 occlusion; therefore, we advocate for performing arterial reconstruction in all M2 complex aneurysm cases in which M2 sacrifice is needed. The types of arterial reconstruction for type B aneurysm cases that we used in this study included STA-RA-M3, STA-M3 bypass, M3-M3 side-to-side or M2M2 in-situ anastomosis [16].

Great effort must be made to preserve the adjacent arterial branches during aneurysm dissection, including the lenticulostriate perforators. For a giant aneurysm with an intraluminal thrombus, opening the aneurysm dome and removing the thrombus with an ultrasonic aspirator may be necessary to ensure decompression, proper clip placement and closure of the bladders. Bleeding of the aneurysm during thrombus resection is usually not severe and can be controlled by applying pressure with a cottonoid patty or by temporary occlusion of the proximal parent artery. Finally, the aneurysm can be trapped or resected.

Complex aneurysms originating from the M1-M2 bifurcation often present as a fusiform morphology, and no obvious neck can be identified. However, one branch of the M2 segment can be preserved by using the constructive clipping strategy. The blood flow through the other M2 segment that is being sacrificed should be reconstructed. The surgical principle is similar to that of a type B aneurysm.

For type $\mathrm{C}$ aneurysms, which originate from the $\mathrm{M} 3$ or greater distal segments, obliteration of the aneurysm with parent artery sacrifice is tolerable in most cases. In certain cases in which the aneurysms originate from the central arteries or angular arteries, acute occlusion of these parent arteries may lead to postoperative neurological deficits. Low flow bypasses, such as an STA-M3 bypass or M3-M3 in-situ anastomosis, are required before aneurysm trapping or resection. For other cases, the necessity of arterial reconstruction depends on the result of intraoperative SSEP and MEP monitoring [6], which is routinely used during our MCA aneurysm surgeries. The changes in SSEP or MEP during the 20-min parent artery occlusion indicate arterial reconstruction; otherwise, we occlude the parent artery directly.

Predicting the tolerance of parent artery sacrificing and guiding the choice of flow replacement

In this series of cases, we mainly relied on the classification and intraoperative MEP and SSEP monitoring to predict the tolerance of parent artery sacrificing. In the past 2 years, we have used quantitative MR angiography (qMRA), which is implemented with Non-invasive Optimal Vessel Analysis (NOVA) software (VasSol Inc., Chicago, IL., USA) to quantitate the blood flow of the affected arteries on which we plan to perform bypasses pre- and intra-operatively. In conjunction with intraoperative MEP and SSEP monitoring, we found this technique would be more accurate to predict the tolerance of parent artery sacrificing (data not shown). Amin-Hanjani et al. reported a series of 23 aneurysmal cases treated with flow replacement, showing that direct intraoperative flow measurement with an ultrasonic flow probe not only helped to guide the choice of flow replacement graft, but also confirmed the adequacy of bypass. In some cases, quantitating the flow deficit is a more accurate way to reveal the flow required of affected arteries after parent artery sacrificing and helps to determine the appropriate revascularization strategy. [3].

\section{Avoidance of main complications}

The main postoperative complications include cerebral ischemia related to occlusion or inadequacy of the bypass graft and injury to the perforators. Gentle operative manipulation to prevent mechanical trauma of the graft, avoid graft spasm by adventitial papaverine irrigation and administer perioperative antiplatelet therapy are among the key methods used to prevent these complications. Avoiding ischemia in the internal capsule or basal ganglion related to lenticulostriate artery occlusion required meticulous intraoperative aneurysm dissection and perioperative administration of antiplatelet therapy and calcium channel blocker agents. Mild hypothermia or the usage of mannitol, dexamethasone, a barbiturate or propofol during the 
temporary occlusion of the parent artery helps to reduce anastomosis-related ischemia. In some type A aneurysm cases, if complete aneurysm obliteration cannot be achieved using ICA occlusion, proximal M1 coiling must be conducted after a delay. This staged M1 occlusion will be more tolerable for patients and help prevent ischemic infarction near the MCA.

\section{Summary}

In this study, we analyzed the surgical modalities, techniques and strategies for treating 59 complex MCA aneurysms in an 8year period. The surgical modalities included direct or reconstructive clipping of the neck, indirect aneurysm occlusion consisting of trapping or resecting the aneurysm, trapping or resecting the aneurysm with EC or IC bypass, and ICA sacrifice with EC-IC bypass. Preoperative analysis of the morphology of the aneurysm, vascular anatomy and the hemodynamic characteristics of each case, intraoperative MEP and SSEP monitoring and cerebral blood flow measurement of the affected arteries were among the key factors in choosing the appropriate surgical modalities and strategies. Thus, we developed a new classification of complex MCA aneurysms based on angioarchitecture. In conjunction with intraoperative electrophysiological monitoring and blood flow measurement techniques, this new classification would help to design an individualized surgical modality and achieve a favorable outcome in each case.

Acknowledgments We thank Xi Chen, MD, for patient database input.

\section{Conflicts of interest None}

Grants This project was sponsored by the National Natural Science 550078 Foundation of China (project no. 30973105 to Wei Zhu), Ministry of Health of the People's Republic of China (project no. 2011BAI08B06 to Ying Mao) and Shanghai Hospital Developing Center Grant (project no. SHDC12010118 to Ying Mao).

Open Access This article is distributed under the terms of the Creative Commons Attribution License which permits any use, distribution, and reproduction in any medium, provided the original author(s) and the source are credited.

\section{References}

1. Alaraj A, Ashley WJ, Charbel FT, Amin-Hanjani S (2008) The superficial temporal artery trunk as a donor vessel in cerebral revascularization: benefits and pitfalls. Neurosurg Focus 24:E7

2. Alaraj A, Ashley WJ, Charbel FT, Amin-Hanjani S (2008) The superficial temporal artery trunk as a donor vessel in cerebral revascularization: benefits and pitfalls. Neurosurg Focus 24:E7

3. Amin-Hanjani S, Alaraj A, Charbel FT (2010) Flow replacement bypass for aneurysms: decision-making using intraoperative blood flow measurements. Acta Neurochir (Wien) Jun 152(6):10211032, discussion 1032

4. Babbu DR, Sano H, Kato Y, Arabi O, Karagiozov KL, Yoneda M, Imizu S, Watanabe S, Oda J, Kanno T (2006) The "Multi Clip" method in unrupture complex middle cerebral artery aneurysms-a case series. Minim Invasive Neurosurg 49:331-334

5. Bederson JB, Zabramski JM, Spetzler RF (1992) Treatment of fusiform intracranial aneurysms by circumferential wrapping with clip reinforcement. Technical note. J Neurosurg 77:478-480

6. Chen L, Lang L, Zhou L, Song D, Mao Y (2012) Bypass or not? Adjustment of surgical strategies according to motor evoked potential changes in large middle cerebral artery aneurysm surgery. World Neurosurg 77:391-398

7. Choi IS, David C (2003) Giant intracranial aneurysms: development, clinical presentation and treatment. Eur J Radiol 46:178-194

8. Drake CG, Peerless SJ (1997) Giant fusiform intracranial aneurysms: review of 120 patients treated surgically from 1965 to 1992 . J Neurosurg 87:141-162

9. Gomes F, Dujovny M, Umansky F, Ausman JI, Diaz FG, Ray WJ, Mirchandani HG (1984) Microsurgical anatomy of the recurrent artery of Heubner. J Neurosurg 60:130-139

10. Gomes FB, Dujovny M, Umansky F, Berman SK, Diaz FG, Ausman JI, Mirchandani HG, Ray WJ (1986) Microanatomy of the anterior cerebral artery. Surg Neurol 26:129-141

11. Kimura T, Nishimura K, Fukaya S, Morita A (2010) Fusiform aneurysm of the anterior communicating artery treated by vascular reconstruction: case report. Neurosurgery 66:E1025-E1026

12. Lanzino G, Spetzler RF (2003) Clip wrapping for partial avulsion of the aneurysm neck. Technical note. J Neurosurg 99:931-932

13. Nakajima H, Kamiyama H, Nakamura T, Takizawa K, Tokugawa J, Ohata K (2012) Direct surgical treatment of giant middle cerebral artery aneurysms using microvascular reconstruction techniques. Neurol Med Chir (Tokyo) 52:56-61

14. Rodriguez-Hernandez A, Sughrue ME, Akhavan S, HabdankKolaczkowski J, Lawton MT (2013) Current management of middle cerebral artery aneurysms: surgical results with a "clip first" policy. Neurosurgery 72(3):415-427. doi:10.1227/NEU.0b013e3182804aa2

15. Romani R, Lehto H, Laakso A, Horcajadas A, Kivisaari R, von Und ZFM, Niemela M, Rinne J, Hernesniemi J (2011) Microsurgery for previously coiled aneurysms: experience with 81 patients. Neurosurgery 68(140-153):153-154

16. Sanai N, Zador Z, Lawton MT (2009) Bypass surgery for complex brain aneurysms: an assessment of intracranial-intracranial bypass. Neurosurgery 65(670-683):683

17. Sano H (2010) Treatment of complex intracranial aneurysms of anterior circulation using multiple clips. Acta Neurochir Suppl 107:27-31

18. Seo BR, Kim TS, Joo SP, Lee JM, Jang JW, Lee JK, Kim JH, Kim SH (2009) Surgical strategies using cerebral revascularization in complex middle cerebral artery aneurysms. Clin Neurol Neurosurg 111:670-675

19. Sughrue ME, Saloner D, Rayz VL, Lawton MT (2011) Giant intracranial aneurysms: evolution of management in a contemporary surgical series. Neurosurgery 69(1261-1270):1270-1271

20. Sughrue ME, Saloner D, Rayz VL, Lawton MT (2011) Giant intracranial aneurysms: evolution of management in a contemporary surgical series. Neurosurgery 69(1261-1270):1270-1271

21. van Doormaal TP, van der Zwan A, Verweij BH, Han KS, Langer DJ, Tulleken CA (2008) Treatment of giant middle cerebral artery aneurysms with a flow replacement bypass using the excimer laser-assisted nonocclusive anastomosis technique. Neurosurgery 63(12-20):20-22

22. Waldron JS, Halbach VV, Lawton MT (2009) Microsurgical management of incompletely coiled and recurrent aneurysms: trends, techniques, and observations on coil extrusion. Neurosurgery 64(301-315):315-317

23. Yang I, Lawton MT (2008) Clipping of complex aneurysms with fenestration tubes application and assessment of three types of clip techniques. Neurosurgery 62:S371-S378, 378-379 